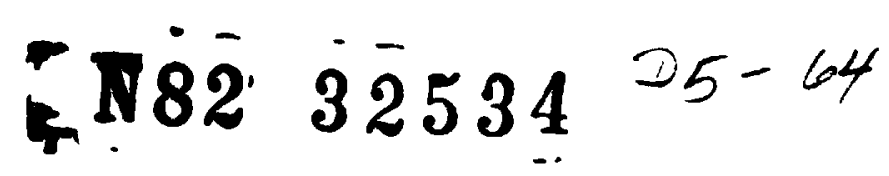

TDA Progress Report $42-70$

Mey end dure 1892

\section{A Structure Function Representation Theorem With Applications to Frequency Stability Estimation}

\author{
C. A. Greenhall \\ Communications Systerns Research Section
}

\begin{abstract}
Random processes with stationary nth differences serve as models for oscillator phase noise. The theorem proved here allows one to obtain the structure function /covariances of the nth differences) of such a process in terms of the differences of a single function of one time varinhle. In turn, this function can easily be obtained from the spectral density of the process. The theorem is used for computing the variance of two estimators of frequency stability.
\end{abstract}

\section{Introduction}

Let the output of a precision oscillator be modelled by cos $\left\{2 \pi \nu_{0}[t+x(t)]\right\}$, where $x(t)$ is a random process representing the "phase time" noise. The most widelv used time-doman measure of oscillator stability is the Allan variance, defined by the ensemble average

$$
\sigma_{A}^{2}(\tau)=\frac{1}{2 \tau^{2}} E\left[\Delta_{\tau}^{2} x(s)\right]^{2},
$$

provided that the expectation exists and is independent of the time s. Here, $\Delta_{T}^{2}$ is the backward 2 nd difference operator, given by

$$
\Delta_{\tau}^{2} f(t)=f(t)-2 .(t-\tau)+f(t-2 \tau)
$$

The theorem given here arose from. the desire to compute the performance of estimators of $o_{A}^{2}(\tau)$. Suppose that $x(t)$ is given on an interval $0 \leqslant t \leqslant T$. Fix $\tau \leqslant \Gamma / 2$, and set

$$
\xi(t)=\frac{1}{N^{2}}=\Delta_{r}^{2} x(t)
$$

A class of unbiased estimators of $\sigma_{A}^{2}(\tau)$ is given by

$$
V=\int_{2 \tau}^{T} \xi^{2}(t) w(t) d t
$$

where $w(t)$ is a weighting function (or measure) whose total weight on $[2 \tau, T]$ is 1 . Two members of this class are treate $]$ in Section IV.

Assume further that $\xi(t)$ is a stationary Gaussian process with zero mean and autocovariance function $R_{\xi}(t)$. Then $\xi^{2}(t)$ is a stationary process with mean $\sigma_{A}^{2}(\tau)$ and autocovariance furition $2 R_{\xi}^{2}(t)$. It follows that

$$
E V=E \xi^{2}(t)=\sigma_{A}^{2}(\tau)
$$

$$
\operatorname{Var} V=\int_{2 \tau}^{T} \int_{2 \tau}^{T} 2 R_{\xi}^{2}(s \cdot t) w(s) w(t) d s d t
$$


Evidently, to compute $V a r V$, we need to obtain $R_{\xi}(t)$ from the model for $x(t)$. Let us suppose, temporarily, that $x(t)$ is stationary, with autocovariance function

$$
R_{x}(t)=\operatorname{Cov}[x(s+t), x(s)],
$$

not depending on s. A straightforward computation yields $E \Delta_{?}^{2} x(s)=0$, and

$$
E \Delta_{\tau}^{2} x(s+t) \Delta_{\tau}^{2} x(s)=\delta_{\tau}^{4} R_{x}(t)
$$

where $\delta_{\tau}^{4}=\Delta_{T}^{2} \Delta_{T}^{2}$, the central 4th difference operator, also given by

$$
\delta_{\tau}^{4} f(t)=f(t-2 \tau)-4 f(t-\tau)+6 f(t)-4 f(t+\tau)+f(t+2 \tau)
$$

The left side of (4) is called the 2 nd structure function of $x(t)$ (Refs. 1, 2). Letting $t=0$ in (4), we have

$$
\sigma_{A}^{2}(\tau)=\frac{1}{\tau^{2}}\left[3 R_{x}(0)-4 R_{x}(\tau)+R_{x}(2 \tau)\right]
$$

as pointed out by Barnes (Ref. 3). Consequently, since $\left|R_{x}(t)\right|$ $\leqslant R_{x}(0)=\operatorname{Var} x$.

$$
\begin{aligned}
& \sigma_{A}^{2}(\tau) \leqslant \frac{8 \operatorname{Var} x}{\tau^{2}}, \\
& \sigma_{A}^{2}(\tau) \sim \frac{3 \operatorname{Var} x}{\tau^{2}} \quad(\tau \rightarrow \infty),
\end{aligned}
$$

provided $R_{x}(t) \rightarrow 0(t \rightarrow \infty)$.

This is for stationary $x(t)$. On the other hand, for actual oscillators a behavior like (6) is observed only for small $\tau$, below I s for quartz cry stal oscillators and $100 \mathrm{~s}$ for hydrogen masels. As $\tau$ increases, the measured $\sigma_{A}(\tau)$ decreases to a minimum, then stays constant or increases. Of course, since our measurement times are finite, this observation does not "prove" that $x(t)$ is nonstationary. A stationary process with a huge vanance and a tiny bandwidth would explain what we see. for we would be looking onlv at a small piece of the process. If $T$ were to increase beyond the scale of our observatons, then $\sigma_{A}(\tau)$ would ultimately behave like (6) again. However, if we want to describe the behanor of $x(t)$ on realistic ume intervals, a nunobservable low-frequency cutoff only gets in the way. and eventually has to be driven to zero. It is mathematically easier to use a nonstationary model from the very start.
For modelling oscillator phase noise, it is usually sufficiant to let $x(t)$ belong to the class of processes whose 2 nd ditierences $\Delta_{r}^{2} x(t)$ are stationary. Thir class inchudes the stationary proceses and those with stationary lst differences. Such a process has a two-sided "formal" spectral density $S_{x}(\omega)$, which can have a singularity at $\omega=0$ that is strong enough to make

$$
\int_{-1}^{1} S_{x}(\omega) d \omega=\infty
$$

Nevertheless, we always have

$$
\int_{-\infty}^{\infty} \frac{\omega^{4}}{1+\omega^{4}} S_{x}(\omega) d \omega<\infty
$$

for this class of processes. An example is the power-law spectrum

$$
S_{x}(\omega)=\frac{K}{|\omega|^{\beta}},
$$

where $1<\beta<5$.

A rigorous theory of these processes exists (Ref. 1); basically, it shows that one can plunge ahead with the formalism from stationary processes as long as the integrals converge. For example, the transfer function of the operator $\Delta_{\tau}^{2}$ is $\left(1-e^{-i \omega \tau}\right)^{2}$. Therefore,

$$
\begin{gathered}
E \Delta_{\tau}^{2} x(t+s) \Delta_{\tau}^{2} x(s)=\int_{-\infty}^{\infty} e^{i \omega \tau}\left|1-e^{-i \omega \tau}\right|^{4} \\
s_{x}(\omega) \frac{d \omega}{2 \pi}+c^{2} \tau^{4}
\end{gathered}
$$

The extra term $c^{2} r^{4}$ comes from a frequency drift component $c t^{2} / 2$ in $x(t)$. Letting $t=0$, we obtain the Allan variance (1). By this method, the theoretical Allan variance has been evaluated and tabulated (Ref. 4) for $S_{x}(\omega)=K /|\omega|^{k}, k$ an integer, $0 \leqslant k \leqslant 4$. (For $k \leqslant 1$, a high-frequency cutoff is provided.) Allowing $\ell$ to be nonzero appears to make (8) more difficult to evaluate. Yet, for our estimation problem, we do need the full covariance funcion of the process $\Delta_{r}^{2} x(t)$. One longs for the simplicity of (4), with $R_{x}(t)$ given by the simple Fourier integral

$$
R_{x}(t)=\int_{-\infty}^{\infty} e^{i \omega t} S_{x}(\omega) \frac{d \omega}{2 \pi}
$$


This integral does not exist, however, unless $x(t)$ is stationary (or equal to a quadratic polynomial phus a stationary process).

The theorem to be proved here gives an easily computable replacement for $\boldsymbol{R}_{x}(t)$, valid for all processes with stationary $n^{\text {th }}$ differences. Equation (4) is replaced by

$$
E \Delta_{\tau}^{2} x(s+r) \Delta_{\tau}^{2} x(s)=\delta_{\tau}^{4}[2 \operatorname{Re} C(t)]+c^{2} \tau^{4}
$$

where the (nanunique) function $C(t)$ can be computed by two different methods. Here is the second method: Choose an integer $k$ such that $\omega^{k} S_{x}(\omega)$ is integrable near $\omega=0$. Then let

$$
C(z)=I^{*} \int_{0}^{\infty} e^{i \omega x}(i \omega)^{k} s_{x}(\omega) \frac{d \omega}{2 \pi}
$$

in the upper half-plane $\operatorname{Im} z>0$. The operator $I^{k}$ instructs the user to integrate $k$ times with respect to $z$. One may then allow $z$ to be real.

Formally, all $w$ : are doing is differentiating (9) $k$ times and integrating $k$ times. If one does this correctly, one easily gets valid results for all the power-law oscillator noise models. Although Lindsey and Chie (Ref. 2) give a number of formulas that generalize (5), they have to assume that either the phase $x(t)$ or the frequency $d x / d t$ is stationary. For flicker FM or random walk FM noise, these assumptions are false. Lindsey and Chie do hint at the need for distribution theory in this situation. Although our method has obvious connections to the analytic representation of distributions (Ref. 5), we use only the elementary theory of real and analytic functions to arrive at the main result.

To illustrate the theorem, let us consider 'ne noise called random walk frequency modulation, defiried by $S_{x}(\omega)=$ $K / \omega^{4}$. For this noise, $\sigma_{A}^{2}(\tau)$ is proportional to $\tau$ (as we shall soon see); this kind of Allan variance behavior has been observed in hydrogen maser frequency standards for $\tau>10^{4} \mathrm{~s}$ (Ref. 6). Taking $k=4$ in (11), we have

$$
\begin{aligned}
C(z) & =\Gamma^{4} \frac{K i}{2 \pi z} \\
& =\frac{K i}{2 \pi} \frac{z^{3} \ln z}{6} \quad(\operatorname{lm} z>0),
\end{aligned}
$$

in which $\ln z$ is to be analytic in $\operatorname{Im} z>0$. The function $\left(z^{3} \ln z\right) / 6$ is just a particular solution to the equation $f^{(4)}(z)=$ $1 / z$. Taking $\ln z=\ln |z|+i$ Arg $z$ in the upper half-plane. we get

$$
2 \operatorname{Re} C(t)= \begin{cases}0 & (t>0) \\ -\frac{\pi}{6} t^{3} & (t<0)\end{cases}
$$

This function is nothing like the autocovariance function of a stationary process, yet applying the operator $8 \$$ to it gives the result

$$
\begin{aligned}
E \xi(s+r) \xi(s) & =\frac{1}{2 \tau^{2}} \delta_{T}^{4}(2 \operatorname{Re} C(t)) \\
& =\frac{K T}{12} \cdot \begin{cases}(2-r)^{3}-4(1-r)^{3} & (0<r<1) \\
(2-r)^{3} & (1<r<2) \\
0 & (r>2)\end{cases}
\end{aligned}
$$

(where $r=|r| / \tau$ ), which is a perfectly good autocovariance function. In particular,

$$
\sigma_{A}^{2}(\tau)=E \xi^{2}(s)=\frac{K T}{3}
$$

The resul: (13) can also be derived by expressing $\$(t)$ as the output of a filter acting on white noise. The method given here is easier and applies to more general difference operators. Moreover, as Section III shows, all of the power-law spectral models become equally simple. Previously, the odd powers (the "flicker" models) were more difficult to handle than the even powers.

\section{The Representation Theorem}

A mean-continuous random process $x(t)$ is said to have stationary $n^{\text {th }}$ differences if, for each real $\tau$, the process $\Delta^{n} x(t)$ is stationary (in the wide sense). For such a prusess, define the dc coefficient $c$ by

$$
c=\lim _{T \rightarrow \infty} \frac{1}{T} \int_{0}^{T} \Delta_{1}^{n} x(r) d t
$$

(in mean square).

For applications, we shall assumc that the $n^{\text {th }}$ differenceof $x(t)$ are ergodic, so that $c$ is nonrandom. It can then be shown that

$$
c \tau^{n}=E \Delta_{r}^{n} x(t)
$$




\section{ORIGINAL PAGE IS}

The process $x_{0}(t)=x(t)-c t^{n} / n$ ! equals a polynomial of degree $<n$ plus a mean-zero process. Associated with $x_{0}(t)$ is a (two-sided) nonnegative formal spectral density $S_{x}(\omega)$, from which all the covariances of the $n^{\text {th }}$ differences of $x(t)$ can be obtained. If $x(t)$ is real, then $S_{x}(-\omega)=S_{x}(\omega)$.

In connection with these processes, it is convenient to in applied to a function $f(t)$, gives the result

$$
L f(t)=\sum_{\nu} a_{\nu} f\left(t+t_{\nu}\right)
$$

(a finite sum), where the $a_{v}$ are real. Its transfer function is the trigonometric polynomial

$$
L(i \omega)=\sum_{\nu} a_{\nu} e^{i \omega t}
$$

For example,

$$
\begin{aligned}
& \Delta_{\tau}^{n} f(t)=\sum_{\nu=0}^{n}\left(\begin{array}{l}
n \\
\nu
\end{array}\right)(-1)^{\nu} f(t-\nu \tau), \\
& \Delta_{\tau}^{n}(i \omega)=\left(1-e^{-i \omega \tau}\right)^{n}
\end{aligned}
$$

A difference operator $L$ is said to have order $n$ if its transfer function satisfies

$$
\begin{aligned}
& L^{(n)}(0)=\sum_{\nu} a_{\nu} r_{\nu}^{j}=0(0 \leqslant j<n), \\
& L^{(n)}(0)=\sum_{\nu} a_{\nu}{ }^{*} \neq 0 .
\end{aligned}
$$

Such an operator annihilates all polynomials of degree $<n$, and reduces the degree of other polynomials by $n$, as shown by the computation

$$
\begin{aligned}
L r^{m} & =\sum_{\nu} a_{\nu}\left(t+t_{\nu}\right)^{m}=\sum_{\nu} a_{\nu} \sum_{i=0}^{m}\left(\begin{array}{l}
m \\
j
\end{array}\right) t_{\nu}^{j} t^{m-i} \\
& =\sum_{i=0}^{m}\left(\begin{array}{l}
m \\
j
\end{array}\right) L^{(j)}(0) t^{m-i}=\sum_{i=n}^{\infty}\left(\begin{array}{l}
m \\
j
\end{array}\right) L^{(j)}(0) t^{m-j}
\end{aligned}
$$

$L$ and $M$ have orders $l$ and $m$, and $\Lambda=L M$, then $\Lambda$ has order $l+m$, and

$$
\frac{\Lambda^{(l+m)}(0)}{(l+m) !}=\frac{L^{(I)}(0)}{I !} \frac{M^{(m)}(0)}{m !}
$$

If $L$ is given by (15), then the operator $L^{*}$ (of the same order) is defined by

$$
L^{*} f(t)=\sum_{v} a_{v} f\left(t-t_{v}\right)
$$

As we mentioned, an example of order $n$ is $L=\Delta_{\tau}^{n}$, for which $L^{(n)}(0)=n ! \tau^{n}$. Anoth.r example, for $n=2$, is the mixed difference operator $\Delta_{,} \Delta_{j}$, which was used for estimating the relative drift rate of a pair of frequency standards (Ref. 7).

\section{We are now set up to give the main result.}

Theorem. Let $x(t)$ be a real process with stationary $n$th differences, nonrandom dc coefficient $c$, and spectral density $S_{x}(\omega)$. Let $L$ and $M$ be real difference operators of order $n$, and let $\Lambda$ be the difference operator $L M^{*}$ of order $2 n$. Then, the mixed second moments of the processes $L x(t)$ and $M x(t)$ can be put into the form

$$
E L x(s+t) M x(s)=\Lambda\left[(-1)^{n} \frac{c^{2} t^{2 n}}{(2 n) !}+2 \operatorname{Re} C(t)\right]
$$

where the (nonunique) function $\boldsymbol{C}(2)$ is analytic in $\operatorname{lm} z>0$, continuous in $\operatorname{Im} z \geqslant 0$, and can be obtained by either of the following recipes:

Recipe 1. Choose an integer $k$ between 0 and $2 n$ such that

$$
\int_{0}^{-1} \omega^{k} S_{x}(\omega) \mathrm{d} \omega<\infty
$$

Let $C(z)=C_{0}(2)$, where

$$
C_{0}(z)=\int_{0}^{\infty}\left[e^{i \omega z}-\frac{1}{1+\omega^{2 n}} \sum_{i=0}^{k-1} \frac{(i \omega z)}{j !}\right] S_{x}(\omega) \frac{d \omega}{2 \pi}
$$

$(\operatorname{Im} z>0)$ 


\section{ORIGINAL PAGE IS \\ OF POOR QUALTY}

\section{Recipe 2. Choose $k$ as in Recipe 1. Define}

$$
B(z)=\int_{0}^{\infty} e^{i \omega z}(i \omega)^{k} S_{x}(\omega) \frac{d \omega}{2 \pi}(\operatorname{lm} z>0)
$$

Let $C(z)$ be any $k$ th integral of $B(z)$ on $\operatorname{Im} z>0$ (in othet words, any function such that $\left.C^{(k)}(z)=B(z)\right)$. Extend $C(z)$ to the real line by continuity. (This is always possible.)

Remark. The first term in the brackets in (18), when lcted upon by $\Lambda$, becomes

$$
c^{2} \frac{L^{(n)}(0)}{n !} \frac{M^{(n)}(0)}{n !}=E[L x(t)] E[M x(t)]
$$

Corollary. Let $x(t)$ have stationary $n$th differences. The structure function

$$
D(t, \tau)=E \Delta_{\tau}^{n} x(s+t) \Delta_{\tau}^{n} x(s)
$$

of the process $x(t)$ can be obtained from a function of one variable, namely $2 \operatorname{Re} C(t)$, by

$$
D(t ; \tau)=c^{2} \tau^{2 n}+(-1)^{n} \delta_{\tau}^{2 n}(2 \operatorname{Re} C(t))
$$

where

$$
\delta_{T}^{2 n}=\left(\delta_{T}^{2}\right)^{n}=\left(-\Delta_{T} \Delta_{-T}\right)^{n}
$$

the central difference operator of order $2 \boldsymbol{n}$.

These results show that $2 \operatorname{Re} C(t)$ contains the same information as $S_{x}(\omega)$ about the process $x(t)$. We can regard 2 Re $C(t)$ as an analog of the covariance function of a stationary process.

\section{Examples for $\boldsymbol{n}=\mathbf{2}$}

Oscillator phase noise is often modelled by a linear com. bination of independent power-law noises with spectra

$$
\frac{1}{\omega^{4}} \cdot \frac{1}{|\omega|^{3}} \cdot \frac{1}{\omega^{2}} \cdot \frac{F\left(\omega / \omega_{n}\right)}{|\omega|} \cdot F\left(\omega / \omega_{n}\right)
$$

where $F\left(\omega / \omega_{h}\right)$ is an integiable low-pass power response functuon whose one-sided noise bandwidth is $\omega_{h} /(2 \pi)$. Examples are the sharp cutoff ( 1 for $|\omega|<\omega_{h}, 0$ elsewhere) and the exponential cutoff $\exp \left(-|\omega| \omega_{h}\right)$. The above noises are called random walk $F M$, ficker $F M$, whire $F M$, ficker $P M$, and white $P M$. (The term "white" will be used even if $F$ is not exactly flat near zero frequency.)

The introduction has shown how to apply the representation theorem to random walk FM; let us proceed to the calculation of $2 \operatorname{Re} C(t)$ for the other noises. In order to express the results in the established notation of the frequency and timing lierature (Ref. 4), we use the form

$$
S_{x}(\omega)=K_{\alpha}|\omega|^{\alpha-2}, K_{\alpha}=\frac{h_{\alpha}}{2(2 \pi)^{\alpha}}
$$

where a high-frequency cutoff $F\left(\omega / \omega_{h}\right)$ is also applied when $\alpha \geqslant 1$ (PM noises).

First, let $\alpha=0,-1$, or -2 (white, flicker, or randum walk FM). Using Recipe 2 of the theorem with $k=2-\alpha$, we have

$$
B(z)=K_{\alpha} \int_{0}^{\infty} e^{i \omega z} i^{*} \frac{d \omega}{2 \pi}=\frac{K_{a} i^{k+1}}{2 \pi z} \quad(\operatorname{Im} z>0)
$$

Since a $k$ th integral of $1 / z$ is $\left(z^{k-1} \ln z\right) /(k-1)$ !, we get

$$
C(z)=\frac{K_{a} i^{k+1}}{2 \pi} \frac{z^{k-1}}{(k-1) !} \ln z \quad(\operatorname{Im} z \geqslant 0)
$$

in which the branch of $\ln z$ must be analytic in the upper half-plane. We shall let $\ln t$ be real for $t>0$. Let us examine the three cases separately.

White FM: $\alpha=0, k=2$.

$$
C(z)=\frac{K_{0}}{2 \pi i} z \ln z,
$$

$$
C(t)=\frac{K_{0} t}{2 \pi i} \cdot \begin{cases}\ln t & (t>0) \\ \ln |t|+\pi i & (t<0),\end{cases}
$$

$$
2 \operatorname{Re} C(t)=\frac{h_{0}}{2} \cdot \begin{cases}0 & (t>0) \\ t & (t<0) .\end{cases}
$$


Flicker FM: $\alpha=-1, k=3$

$$
\begin{aligned}
C(z) & =\frac{K_{-1}}{4 \pi} z^{2} \ln z, \\
2 \operatorname{Re} C(t) & =\frac{h_{-1}}{2} t^{2} \ln |z| .
\end{aligned}
$$

Random walk FM. $\alpha=-2, k=4$.

$$
\begin{aligned}
C(z) & =\frac{K_{-2} i}{2 \pi} \frac{z^{3}}{6} \ln z, \\
2 \operatorname{Re} C(t) & =\frac{k_{-2} \frac{\pi^{2}}{3}}{3} \cdot \begin{cases}0 & (t \geqslant 0) \\
-t^{3} & (t \leqslant 0) .\end{cases}
\end{aligned}
$$

In a sense, the flicker caso is easier than the others hecause we don't have to keep track oi the imaginary part of $\ln z$.

For handling the PM noises, the exponential cutoff is easier to use than the sharf cutoff, and nay even be more realistic. Let $S_{x}(\omega)=K_{\alpha}|\omega|^{\alpha-2} \exp \left(|\omega|^{\prime}\left(\omega_{h}\right)\right.$, where $\alpha=1$ or 2 . Again letting $k=2-\alpha$, we get

$$
B(z)=\frac{K_{\alpha} i^{k+1}}{2 \pi} \frac{1}{z+i / \omega_{h}} .
$$

Flicker PM: $\alpha=1, k=1$.

$$
\begin{gathered}
C(z)=-\frac{K_{1}}{2 \pi} \ln \left(z+i / \omega_{h}\right), \\
2 \operatorname{Re} C(t)=-\frac{h_{1}}{8 \pi^{2}} \ln \left(t^{2}+1 / \omega_{h}^{2}\right) .
\end{gathered}
$$

For the sharp high-frequency cutoff, $2 \operatorname{Re} C(t)$ turns out to be 2 cosine integral.

White $P M . \alpha=2, k=0$.

$$
2 \operatorname{Re} C(t)=\frac{h_{2} \omega_{h}}{8 \pi^{3}\left(1+\omega_{h}^{2} t^{2}\right)},
$$

which is just $R_{x}(t)$, because now $x(t)$ is stationary.

Fractional noises. It is well known (Ref. 4) that if the spectrum satisfies a power law $S_{x}(\omega)=K_{\alpha}|\omega|^{\alpha-2}$, where -3 $<\alpha<1$, then the Allan variance satifies another power law $\sigma_{A}^{2}(\tau)=$ const $\cdot \tau^{\mu}$, where $\mu=-1-\alpha$. When Allan variance measurements are made, fractional values of $\mu$ sometimes appear over a certain range of $\tau$. Thus, we ought to show how to use the representation theorem for fractional values of $\alpha$ in the range $-3<\alpha<1$. We shall do this for $0<\alpha<1$, leaving the other cases as an exercise for the reader. Taike $k=1$. Then

$$
\begin{aligned}
B(z) & =\frac{K_{\alpha} i}{2 \pi} \int_{0}^{\infty} e^{i \omega z} \omega^{\alpha-1} d \omega \\
& =\frac{K_{\alpha} i}{2 \pi} \frac{\Gamma(\alpha)}{(-i z)^{\alpha}}, \\
C(z) & =i \int B(z) d(-i z)=-\frac{K_{\alpha} \Gamma(\alpha)}{2 \pi(1-\alpha)}(-i z)^{1-\alpha},
\end{aligned}
$$

where the power functions are analytic in the right haif-plane and positive on the positive real axis. Then

$$
2 \operatorname{Re} C(t)=-\frac{h_{\alpha} \Gamma(\alpha) \sin \left(\frac{1}{2} \pi \alpha\right)}{(2 \pi)^{1+\alpha}(1-\alpha)}|t|^{1-\alpha},
$$

As $\alpha \rightarrow 0$, this expression tends to $-h_{0}|t| / 4$, which is indeed a valid representative of $2 \operatorname{Re} C(t)$ for $\alpha=0$.

\section{The Variance of Two Allan Variance Estimators}

Let us return to the estimation problem mentioned in the introduction. Recall that phase time $x(t)$ is given for $0<t$ $<T$. Therefore. $\xi(r)=\Delta_{\tau}^{2} x(t) /(i \sqrt{2})$ is available for $2 \tau<t$ $\leqslant T$. Set $m=T / \tau$. Two unbiased estimators of the Allan variance (1) are

$$
C(z)=B(z)=\frac{K_{2},}{2 \pi} \frac{1}{z+1 / \omega_{n}} .
$$

$$
V_{\tau}=\frac{1}{m-1} \sum_{i=2}^{m} \xi^{2}(j \tau)
$$


( $m$ an integer $>2$ ), called the 7 -overlap estimator, and

$$
V_{0}=\frac{1}{-(n-2)} \int_{2 r}^{m t} \xi^{2}(t) d t
$$

( $m$ real, $>2$ ), called the continuous estimator, which, although it cannot be achieved in practice, represents a limiting case for a sample time $\tau_{0}$ much less than $\tau_{\text {. The use of such an }}$ estimator was suggested by Ho* c. Allan, and Barnes (Ref. 8).

Although the T-overlap estimsicn has been used for many years, it is reasonable to ask whether the continuous estimator has a smaller variance. In other words, ii $\tau_{0} \ll \tau$, should we average all the available samples $\xi^{2}\left(j \tau_{0}\right)$, or should we use only the samples $\xi^{2}(j r)$ ? Since the data collection time $T$ may be weeks or months, this question is more than academic.

The answer depends both on $m$ and on the spectrum of the phase noise. Assume that $x(t)$ is a Gaussian process with stationary 2 nd differences and zero dc component $c$. If we know $S_{x}(\omega)$, then we can compute $C(z)$. By the corollary, the autocovariance function of the mean-zero process $\xi(t)$ is

$$
R_{E}(t)=\frac{1}{2 \tau^{2}} \delta_{\tau}^{4}(2 \operatorname{Re} C(t))
$$

As we said in the introduction, the autocovariance function of the stationary process $\xi^{2}(t)$ is $2 R_{\xi}^{2}(t)$. The means and variances of $V_{T}$ and $V_{0}$ are now computed straightforwardly:

$$
\begin{gathered}
E V_{T} \quad E V_{0}=R_{\xi}(0)=\sigma_{A}^{2}(\tau), \\
\operatorname{Var} V_{\tau}=\frac{2}{(m-1)^{2}} \sum_{i=-m+2}^{m-2}(m-1-|j|) R_{\xi}^{2}(j \tau), \\
\operatorname{Var} V_{0}=\frac{2}{T_{2}^{2}} \int_{-T_{2}}^{T_{2}}\left(T_{2}-|t|\right) R_{\xi}^{2}(t) d t,
\end{gathered}
$$

where $T_{2}=(m-2) \tau$. (Of course, we recognize that $R_{\xi}(t)$ is an even function.)

The computations have been carried out for white FM, flicker FM, and random walk FM (see Section III), all of whicls have been observed in actual oscillators. The flicker FM results were computed numerically; the others are in closed form, which, however, we shall not give here. For white FM and random walk FM, the r.overlap formulas agree with those of Lesage and Audoin (Ref. 9); for flicker FM, the T-overlap numbers agree with Yoshimura's (Ref. 10).

The results are presented in terms of "degrees of freedom, defined for a positive estimator $V$ by

$$
\text { d.f. }=\frac{2(E V)^{2}}{\operatorname{Var} V}
$$

Given d.f., one sometimes uses the appropriate chi-squared distribution for constructing confidence intervals about the estimate (Ref. 8). Whether or not this is done, the d.f. remains a useful figure of merit.

In Fig. 1, for the above ihre. noise types, we plot d.f./ $(m-1)$ vs $m$ for $V_{T}$ and $V_{0}$. For white FM, $V_{0}$ is always better than $V_{7}$. For flicker FM, $V_{0}$ is better than $V_{\text {, except for }}$ $m \leqslant 3$. For random walk $F M, V_{t}$ is better than $V_{0}^{\top}$ for $m \leqslant 18$. Of course, the smaller values of $m$ are more critical, since d.f. is roughly proportional to $m-1$.

It may seem paradoxical for $V_{r}$ to be better than $V_{0}$, since $V_{0}$ uses all the available data. Both estimators are special cases of (2), however; if one looks for the optimal (minimal variance) estimator of the class (2) (for a given noise type), one will probably find that the optimal weighting function $w(t)$ is noncoustant and almost everywhere nonzero. In other words, one should use all the data, but in a nonuniform way.

\section{Concluding Summary}

Oscillator stability is usually characterized by the behavior of $n$th order differences of the phase. The theoretical evalustion, from ihe phase noise spectrum, of the variances and covariances of these differences involves messy trigonometric integrals, such as (9). The messiness is caused by a ( $2 n)$ th order difference operator tangled up inside the integral. Our representation theorem breaks the integral evaluation into two easy steps. (1) evaluation of a much simpler integral depending only on the noise spectrum; (2) application of that sume difference operator to the result of step (1).

In effec, the evaluation of these integrals is uncoupled into two independent operations. In Section III, we tabulated only the result of $\operatorname{step}(1)$ (the function called $2 \operatorname{Re} C(t)$ ) for all the usual powcr-law oscillator noise models. This short "onedimensional" table, plus another one-dimensional table of dif. ference operators, can generate a two-dimensional table of resulis as found, for example, in Ref. 4. 
As an application, we expmined two Allan rariance estjmators Because the representation theorem delivers such simple closed forms for the required autocovariance functions, the computations were quickly executed by a simple BASIC program. The theorem can slso be used for evailiating the performance of frequency drift estimators. In general, we get an estimator of frequency drift rate (the dc coefficient $c$ ) by operating upon oscillator phase with a second-order difference opp-stios. $L$ that need not be of form $\Delta_{T}^{2}$. Hen, the full generality of the theorem is needed.

\section{References}

1. Yaglum, A. M., "Correlation theory of processes with random stationary nth increments," Amer. Math. Soc. Translations, Ser. 2, Vol 8, pp. 87.141, 1958.

2. Lindsey, W. C., and Chie, C. M., "Theory of oscillator instability based upon structure functions," Proc. IEEE, Vol. 64, pp. 1652-1666, 1976.

3. Barnes, J. A., "Atomic timekeeping and the statistics of precisiun signal generators," Proc. IEEE, Vol. 54, pp. 207-220, 1966.

4. Barnes, J. A., et al., "Characterization of irequency stability," IEFE Trans. Instrum. Meas., Vol. IM-20, pp. 105.120, 1971.

5. Bremermann, H., Distributions, Complex Variables, and Turier Transforms, Addison-Wesley, Reading, Mass., 1965.

6. Kuhnie, P. F., "Hydrogen maser 'mplenentation in the Deep Space Network at the Jet Propulsion Laboratory," Proc. IIth Ann. Prec ise Time and Time Interval Applications and Planning Mecting, Goddard Space Flight Center, Greenl , Md., 1979.

7. Greenhall, C. A., "Removal of drift from frequency stability measurements, 'TDA Progriss Report 42-65, pp. 127-132, Jet Propulsion Laboratory, Pasadena, $\mathrm{Ca}^{14}$.., Oct. 15, 1981.

8. Howe, D. A., Allan, D. W., and Barnes, J. A., "Properties of signal sources and measurement methods," Proc. 35th Ann. Symp. on Frequency Control, USAERADCOM, Fort Monmouth, N.J.. pp. Al-A47, 1981.

9. Lesage, P., and Audoin, C., "Estimation of the two-sample variance with limited number of data," Proc. 31st Ann. Symp. on Frequency Control, pp. 311-313, 1277.

10. Yoshimura, k., "Characterization of fren"ency stat" "',: uncertainty due to the autocorrelation of the frequency fluctuations," IEEE Tims. Instrum Meas., Vol. IM-27. pp. 1.7, 1978. 
CRIGINAL PRGE 13
OF POOR QUALITY

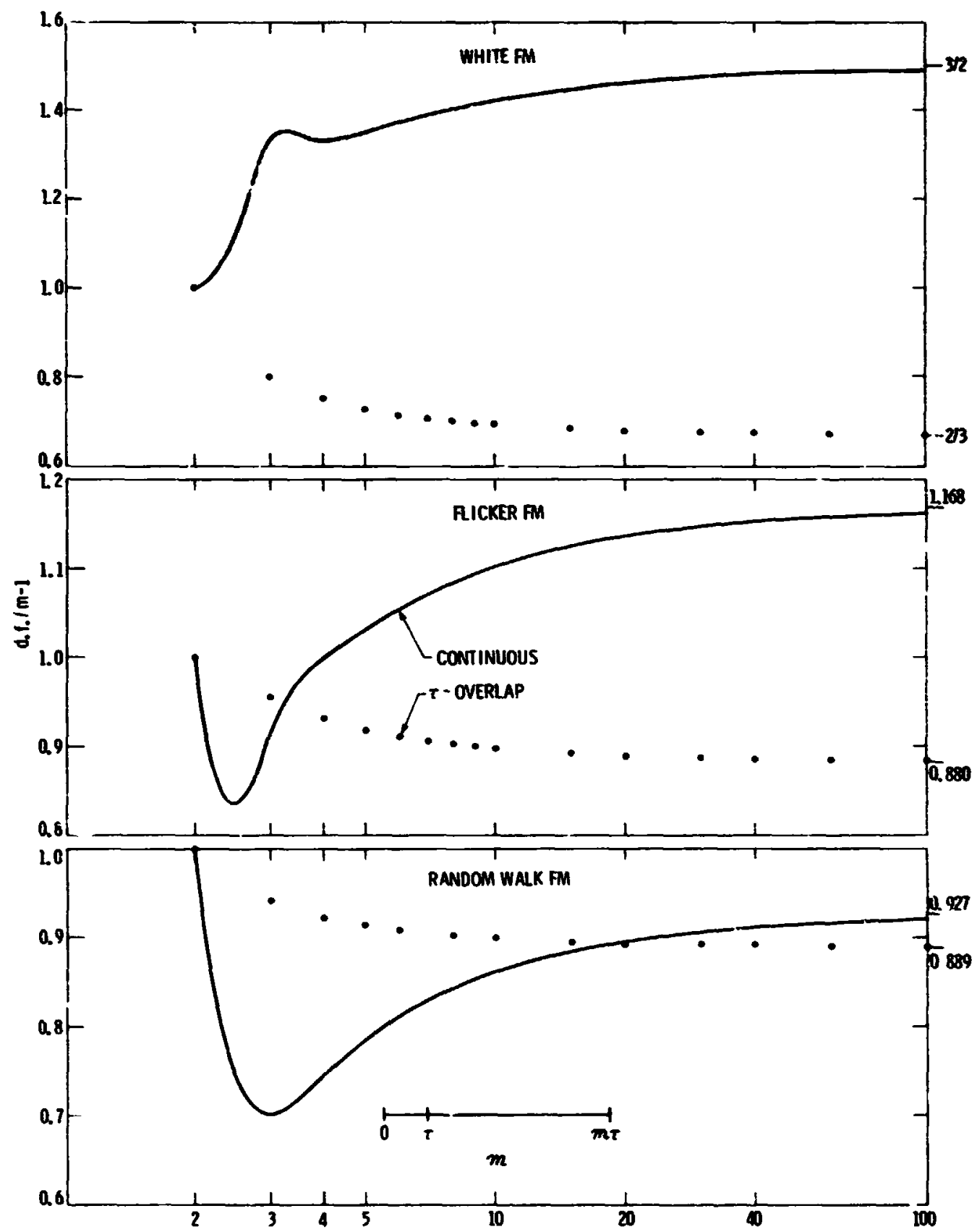

Fig. 1. Degreses of freadom of the ;-overtep and continuous Alien variance ectimaties for white, fileker, and random-walk frequency modulation. The numbers on the right give the hortzortal sesymptotes. The observation time is $\eta_{T}$ 


\section{ORIGNAL PAGE $\mathrm{S}$
OF POOR QUALT'}

\section{Appondx \\ Proof of the Representetion Theorem}

For convenience, let as first set down an elementary estimate of the Taylor remeinder for $e^{x}$ : li $\operatorname{Re} z \leqslant 0, k>1$, then

$$
\left|e^{2}-\sum_{k=0}^{k-1} \frac{z^{j}}{i}\right| \leqslant \frac{\mid z k^{k}}{k !}
$$

This can be obtained from the integral form of the remainder.

In analogy with the usend notation for mixed structure functions $D\left(t ; r_{1}, r_{2}\right)$ (Ref. 1), denote the left side of (18) by $D(t ; L, M)$. Without loss of generaity, we can assume that the dc coefficient $c$ is zero. Begin with the spectral representation of $D(r ; L, M)$ as given by $Y$ aglon.

$$
\begin{aligned}
D(t ; L, M) & =\int_{-\infty}^{\infty} e^{i \omega t} L(i \omega) M(-i \omega) S_{x}(\omega) \frac{d \omega}{2 \pi} \\
& =\int_{-\infty}^{\infty} \Lambda e^{i \omega t} S_{x}(\omega) \frac{d \omega}{2 \pi} \\
& =2 \operatorname{Re} A(t) .
\end{aligned}
$$

where

$$
A(z)=\int_{0}^{\infty} \Lambda e^{i \omega z} S_{x}(\omega) \frac{d \omega}{2 \pi}
$$

for $z=t+i u(u>0)$, and .1 operates on functions of $t$. The name of the game is pulling $\Lambda$ outside the integral. If you do this brutally. the integral usually blows up.

Since the spectral density $S_{x}(\omega)$ always satisfies

$$
\int_{0}^{\infty} \frac{\omega^{2 n}}{1+\omega^{2 n}} S_{x}(\omega) d \omega<\infty
$$

(Ref. 1), the integer $k$ specified in Recipe 1 exists. W: can alway: take $k=2 n$; it is often possible and desirable to use a smaller $k$. If $k=0$ works. then $S_{x}(\omega)$ is integiable, $\Lambda$ comes outside the integral in (A3), and we are done. In this case. $Q z)=A(z)$. and $x(y)$ is a poly nominal plus a stationary process whose autocovartance function is $2 \operatorname{Re} C(t)$.

Assume $d>1$, Sin 2 , for all $u>0$.

$$
\int_{0}^{\infty} e^{-\omega \omega} \omega^{*} S_{x}(\omega) d \omega<\infty \text {, }
$$

the function $S(z)$ of Recipe 2 exists and is andytic in Im $z>0$. Let us now look at $C_{0}(z)$ as given in Recipe 1 . It will soon be stocon that

(a) $C_{0}$ (z) is continuous on $\mathrm{Im},>0$ and analytic on $\operatorname{Im} z>0$;

(b) $C_{d}^{(k)}(2)=B(2)$ for $\operatorname{Im} 2>0$;

(c) $\Lambda C_{0}(z)=A(z)$ for $\operatorname{Im} z>0$.

Assuming (a) - (c), we see that $C_{0}(z)$ is a $k^{\text {th }}$ integral of $B(z)$ on $\operatorname{Im} z>0$; any uther $k^{\text {th }}$ integal $\alpha(z)$ differs from $C_{0}(z)$ by at most a polyncmial of degree $<k$. Since $A$ annihilates all poiynomials of degree $<2 n$, properties (a) - (c) bold with $C_{0}(z)$ replaced by $Q(z)$, ant the theorem follows from (A2).

To prove (a) - (c) denoted the bracketed expression in (19) by $E(z, \omega)$, which splits into the two parts

$$
E_{1}(z, \omega)=\frac{1}{1+\omega^{2 n}}\left[e^{i \omega z}-\sum_{i=0}^{k-1} \frac{(i \omega z)^{j}}{j !}\right],
$$

$$
E_{2}(z, \omega)=\frac{\omega^{2 n}}{i+\omega^{2 n}} e^{i \omega z}
$$

Accordingly. $C_{0}(z)$ splits into two integrais $C_{1}(z)$ and $C_{2}(z)$. Since $C_{2}(z)$ is the Fourier transform of an integrable function, $C_{2}(z)$ is continuous on $\operatorname{Im} z>0$; on $\operatorname{Im} z>0$ it is analytic and can be differentiated repeatedly under the integral sign.

Differentiatins, $E_{1}$ repeatedly and applying $(A 1)$, we get the bound

$$
\frac{\partial p}{\partial z^{p}} E_{1}\left(z, \omega_{i}\right) \leqslant \frac{1}{1+\omega^{2 n}} \frac{a^{k-p} \omega^{k}}{(k-p) !}
$$

valid for $0 \leqslant p \leqslant k, \operatorname{Im} z \geq 0,|z| \leqslant a$. The right side of (A4) times $S_{x}(\omega)$ is integrable. Therefore, the function $C_{1}(z)$, on the domain $\operatorname{lm} z>0$, can be differentiated $k$ times under the integral sign. In particular, $C_{1}(z)$ is continuous on tnis domain. This proves (a), and shows that $C_{0}(2)$ can be differentiated $k$ tumes under the integral sign on $\operatorname{Im} z>0$. Doing so gives (b). Property (c) is true because $\Lambda$ annihilates polynomials of degree $k-1$. The theorem is proved. 